\title{
CMOS-compatible wafer-scale Si subulate array for superb switching uniformity of RRAM with localized nanofilaments
}

\author{
Ying Zhang ${ }^{1,2,3}$, Xiaolong Zhao ${ }^{2 *}$, Xiaolan $\mathrm{Ma}^{2}$, Yu Liu ${ }^{1}$, Xuanze Zhou ${ }^{2}$, Meiyun Zhang ${ }^{1}$, \\ Guangwei $\mathrm{Xu}^{2}$ and Shibing Long ${ }^{2}$
}

\begin{abstract}
Resistive switching random access memory (RRAM) is one of the most promising candidates with highdensity three-dimensional integration characteristics for nextgeneration nonvolatile memory technology. However, the poor uniformity issue caused by the stochastic property of the conductive filament (CF) impedes the large-scale manufacture of RRAM chips. Subulate array has been introduced into the RRAM to minimize the CF randomness, but the methods are cumbersome, expensive, or resolution-limited for large-scale preparation. In this work, Si subulate array (SSA) substrates with different curvature radii prepared by a wafer-scale and nanoscale-controllable method are introduced for RRAM fabrication. The SSA structure, which induces a quasi-single CF or a few CFs formed in the tip region (TR) of the device as evidenced by the high-resolution transmission electron microscopy and energy dispersive spectroscopy characterization, dramatically improves the cycle-to-cycle and device-to-device uniformity. Decreasing the curvature radius of the TR significantly improves the device performance, including switching voltages, high/low resistance states, and retention characteristics. The improved uniformity can be attributed to the enhanced local electric field in the TR. The proposed SSA provides a low-cost, uniform, CMOS-compatible, and nanoscale-controllable optimization strategy for the largescale integration of highly uniform RRAM devices.
\end{abstract}

Keywords: resistive switching, memristor, RRAM, Si subulate array, enhancement of electric field

\section{INTRODUCTION}

With the rapidly increasing need for next-generation highdensity nonvolatile memory devices [1], resistive switching random access memory (RRAM) has received enormous attention as a prominent candidate in recent years due to its high density, low power consumption, and fast switching speed [2-5]. The repeated formation and rupture of the conductive filament (CF) correlated to the redox of defects (metal atoms, oxygen vacancies, etc.) are extensively considered to be the dominant mechanism of the resistance switching phenomenon [6-10]. However, because of the intrinsic problem of the stochastic generation/recombination of defects under an external electric field, which is analogous to the well-known dielectric break- down, the generation and rupture of the CF are likewise random. The randomness of CF ultimately induces a serious fluctuation in the switching parameters [11-15]. Significant parameter fluctuations have become the key obstacle hindering the large-scale manufacture of metal-oxide RRAM [7,16,17]. Many methods have been proposed to improve the switching uniformity of RRAM, including inserting metal nanoparticles into the oxide layer [18-20], introducing an interface layer or using a double-layer dielectric [21-23], doping impurities $[24,25]$, or adding an ionic barrier with hollowed channels [26,27].

Nanotip structure has also been introduced into RRAM and proved effective to improve the RRAM uniformity by enhancing the local electric field at the tip. To date, various strategies have been used to fabricate subulate arrays. Template stripping has been demonstrated an effective method to construct active metal pyramids with high-quality tips to facilitate the ionization of metal atoms and fast migration of generated metal ions $[28,29]$. Particularly, anisotropic wet etching has been developed to fabricate a nano-cone Si bottom electrode (BE), which renders the $\mathrm{SiN}_{x}$-device good nonlinear current-voltage $(I-V)$ characteristics [30]. In addition, electron beam-induced deposition has been presented to vary the nanotip geometries [31]. And electron beam lithography has been utilized to shrink the diameter of the pyramid-type electrodes down to sub-100 nm [32]. And the minimum diameter of the nanotip modulated by ion irradiation combined with a replica-molding process on polyethylene terephthalate substrates can reach approximately $50 \mathrm{~nm}$ [33]. However, these methods improve the switching uniformity at the cost of sacrificing the intrinsic RRAM advantages, such as simplicity, low cost, or high-density integration. In addition, the relationship between the curvature radius (c-radius) of the tip region (TR) and device performance has scarcely been the focus of previous studies. To push RRAM towards practicality, methods that improve the uniformity but do not harm the intrinsic advantages of RRAM devices should be urgently developed.

In this work, a series of special Si subulate array (SSA) substrates fabricated by a low-cost, CMOS (complementary metal oxide semiconductor)-compatible, nanoscale-controllable, and wafer-scale process were utilized to configure highly uniform RRAM devices. Compared with the control device with a planar Si substrate, the RRAM devices with subulate substrates exhibit

\footnotetext{
${ }^{1}$ Key Laboratory of Microelectronic Devices \& Integration Technology, Institute of Microelectronics, Chinese Academy of Sciences, Beijing 100029, China

${ }^{2}$ School of Microelectronics, University of Science and Technology of China, Hefei 230026, China

${ }^{3}$ University of Chinese Academy of Sciences, Beijing 100049, China

* Corresponding author (email: xlzhao77@ustc.edu.cn)
} 
significantly improved cycle-to-cycle and device-to-device uniformity, which can be attributed to the enhanced local electric field in the TR. Decreasing the c-radius significantly improves the device performance, including the resistive window, retention characteristics, and uniformity of switching voltages ( $V_{\mathrm{SET}}$ and $V_{\text {RESET }}$ ) and resistance. The SSA substrates enhance the local electric field, control a few CFs formed in the TR of each device, and further optimize the device switching performance. By transmission electron microscopy (TEM) and energy dispersive spectroscopy (EDS) characterization, CFs were demonstrated to be generated in the TR of the subulate device, where the electric field is enhanced by the tip with a small c-radius. The low-cost SSA fabrication process (photolithography, etching, deposition, etc.) is fully compatible with standard CMOS process for largescale integration. The introduced subulate RRAM device with the ability to induce CF formation has great potential in achieving high-uniformity RRAM devices for commercial applications.

\section{EXPERIMENT SECTION}

\section{SSA substrate preparation}

Before the fabrication of the subulate structure, the natural oxide layer of the $\mathrm{p}^{+}-\mathrm{Si}$ substrate was removed by diluted HF (volume ratio of $\left.\mathrm{HF}: \mathrm{H}_{2} \mathrm{O}=1: 7\right)$. After the photolithography process, a $100-\mathrm{nm} \mathrm{HfO}_{2}$ with a $2.5-\mu \mathrm{m}$ diameter protective layer was deposited (radio-frequency magnetron sputtering, RFMS, $0.5 \AA \mathrm{s}^{-1}, 2 \times 10^{-4} \mathrm{~Pa}$ cavity vacuum, room temperature (RT)) onto the substrate as a mask. After a lift-off process to form the pattern, the subulate substrate was prepared by etching the $\mathrm{Si}$ substrate along the $\langle 100\rangle$ crystal orientation by inductively coupled plasma (ICP) etching with $\mathrm{SF}_{6}$ gas (ICP-98A, $400 \mathrm{~W}$ source power, $40 \mathrm{sccm}\left(1 \mathrm{sccm}=\mathrm{m}^{3} \mathrm{~min}^{-1}\right)$ flux of $\mathrm{SF}_{6}$ at $1 \mathrm{~Pa}$ pressure, $0 \mathrm{~W}$ base power). The smallest $\mathrm{c}$-radius was achieved with a critical ICP etching time of $160 \mathrm{~s}$ and the c-radius increased with etching time (beyond the critical time).

\section{RRAM device preparation}

Subulate RRAM devices with one tip in each memory cell were fabricated on the SSA substrates. Firstly, Ti/Pt (5/10 nm) BE was deposited by RFMS at RT. Secondly, $\mathrm{ZrO}_{2}$ resistive switching (RS) layer with a thickness of $10 \mathrm{~nm}$ was prepared on the BE by RFMS with a sputtering rate of $0.13 \AA \mathrm{s}^{-1}$. Thirdly, Ag top electrode (TE) with a thickness of $30 \mathrm{~nm}$ was deposited by RFMS and patterned via the lift-off process. The planar device with a traditional planar structure without tips was prepared as the control sample. The fabrication processes of the planar RRAM device were the same as those used for the subulate devices. The $\mathrm{TE}$ areas of both the subulate and planar devices are $100 \mu \mathrm{m} \times$ $100 \mu \mathrm{m}$.

\section{Characterization}

The direct-current (DC) mode and pulse mode switching characteristics of the devices were implemented in atmosphere using a semiconductor characterization system (Agilent B1500A Semiconductor Device Parameter Analyzer). A waveform generator/fast measurement unit module (WGFUM) was used to simultaneously generate the voltage pulse and measure the response current in this experiment. Images of the morphology of devices were obtained using a field-emission scanning electron microscope (SEM, ZEISS SUPRA 55 SAPPHIRE). The three-dimensional (3D) profiles of the SSA and planar RRAM devices were observed by a $3 \mathrm{D}$ optical microscope. High-resolution TEM (HRTEM) and EDS composition analysis were conducted with a Field Emission Gun/TEM (FEI Tecnai TF-20, UK) operated under $200 \mathrm{kV}$ voltage.

\section{RESULTS AND DISCUSSION}

The fabrication process of the $\mathrm{Ag} / \mathrm{ZrO}_{2} / \mathrm{Pt}$ subulate device is shown in Fig. 1a and a detailed description is provided in the EXPERIMENT SECTION. The process mainly involves (1) the deposition of $\mathrm{HfO}_{2}$ protective layer, (2) etching to form SSA, and (3) configuration of BE, RS layer, and TE. The SSA substrate was prepared via ICP with $\mathrm{SF}_{6}$ gas etching the planar $\mathrm{p}^{+}-\mathrm{Si}$ substrate. The SEM images obtained during the etching process $(100,130$, and $160 \mathrm{~s}$ ) are exhibited in Fig. 1b. The smallest c-radius was achieved with a critical ICP etching time of $160 \mathrm{~s}$, when the $\mathrm{HfO}_{2}$ "cap" dropped out and was blown away by the gas. The c-radius of the TR can be further controlled by prolonging the etching time. Subsequently, $\mathrm{Pt} / \mathrm{ZrO}_{2} / \mathrm{Ag}$ RRAM devices were prepared on the SSA substrates (one tip per cell). The thicknesses of the $\mathrm{BE}$, RS layer, and $\mathrm{TE}$ were 10,10 , and $30 \mathrm{~nm}$, respectively. Fig. 1c-e show SEM images of the subulate RRAM devices with different c-radii, namely, 54, 40, and $25 \mathrm{~nm}$ (S1, S2, and S3). As observed by $3 \mathrm{D}$ optical microscopy, a large-scale and uniform SSA was obtained (Fig. 1f). And the statistical data of c-radius from randomly selected 12 cells for S3 are shown in Fig. S1 (Supplementary information). The main limitation of tip miniaturization is the exposure technology, which can be overcome through the application of electron beam lithography and immersion exposure; thus, the device size can theoretically be scaled down. The fabrication processes of the planar RRAM device were the same as those of the subulate devices (Fig. S2).

The planar and subulate $\mathrm{Pt} / \mathrm{ZrO}_{2} / \mathrm{Ag}$ devices with different cradii were operated under typical bidirectional nonvolatile RS mode with a compliance current $\left(I_{\mathrm{cc}}\right)$ of $500 \mu \mathrm{A}$. The current limit protects the device from hard breakdown during electrical operations. For all electrical measurements, the TE was biased, while the BE was grounded. Fig. 2 presents typical $I-V$ curves of the planar and subulate devices (S1, S2, and S3) from 50 consecutive switching cycles. The subulate RRAM devices exhibit superior SET/RESET uniformity in comparison to the planer device. The RESET process contributes to the rupture of CFs, which is dominated by electrical migration/diffusion of $\mathrm{CF}$ components under electrochemical effects and Joule heating effects. Two different types of RESET behaviors occur in the RRAM devices, namely progressive RESET and abrupt RESET. S2 and S3 with relatively small c-radius exhibit abrupt RESET, whereas planar device and S1 exhibit progressive RESET. The slope of the RESET process is strongly dependent on the CF morphology, including its shape [34], size [35], and quantity [36-38]. A large CF volume results in a decreased low resistance state (LRS) and contributes to a progressive RESET, while a small CF volume causing a higher LRS tends to result in abrupt RESET. Apparently, the LRS of S2 and S3 is smaller than that of planar and S1. This difference can be attributed to the tailored subulate structure, which localizes the electric field distribution and further reduces the CF quantity in the device. The potentially multiple-CF devices (planar and S1) undergo a gradual transition to high resistance state (HRS) as the CFs are ruptured sequentially, while devices with a quasi-single CF or a few CFs (S2 and S3) switch to HRS with a sudden current drop. Overall, 
a
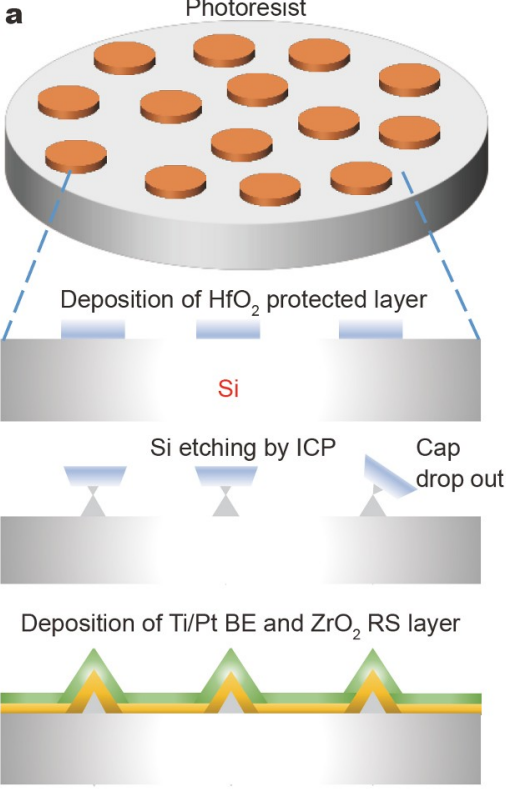

Deposition of Ag TE after patterning

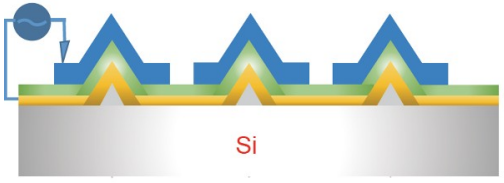

b

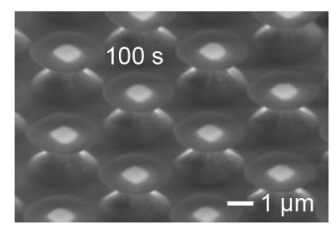

c $\mathrm{S1}$
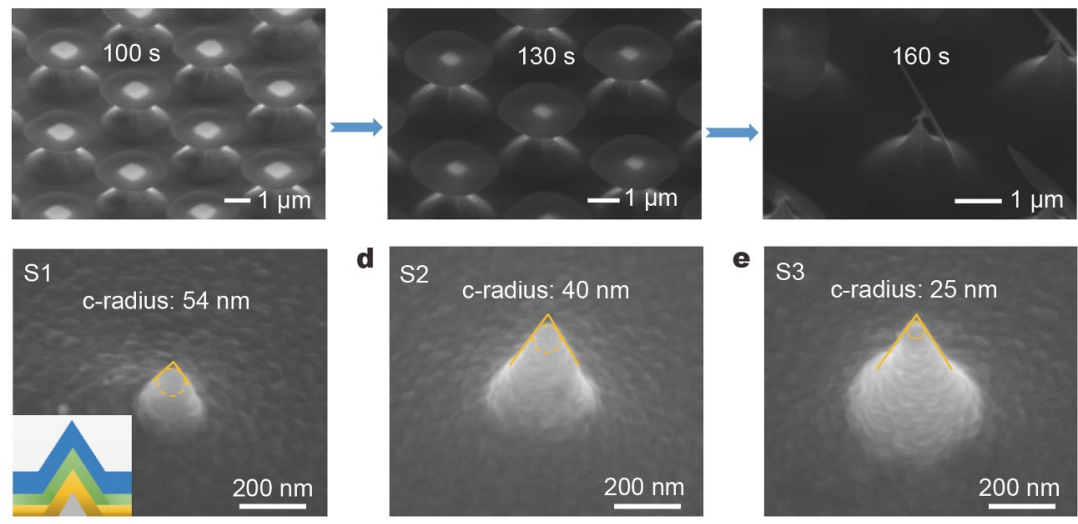

d $\mathrm{S} 2$
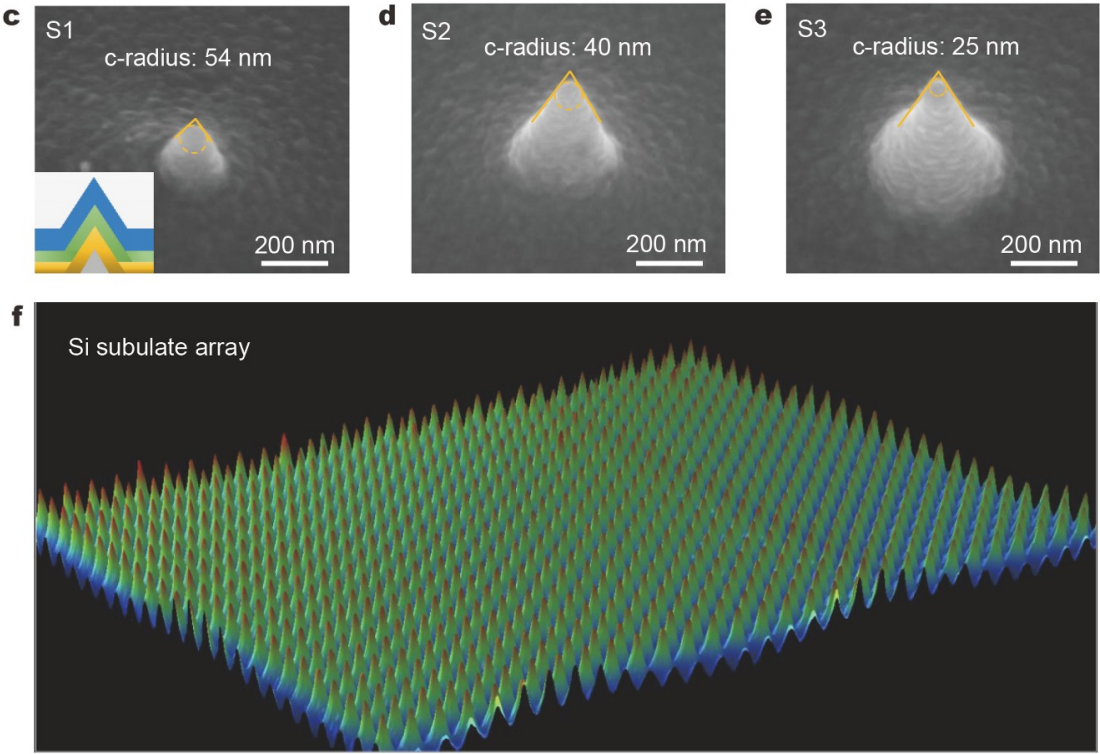

Figure 1 Fabrication of wafer-scale SSA. (a) Schematics of the SSA fabrication process. (b) SEM images of the SSA during the ICP etching process. (c-e) SEM images of the subulate RRAM devices with different c-radii (S1 $54 \mathrm{~nm}, \mathrm{~S} 240 \mathrm{~nm}$, and S3 $25 \mathrm{~nm}$ ). (f) 3D profile of uniform SSA from a typical region of the substrate.
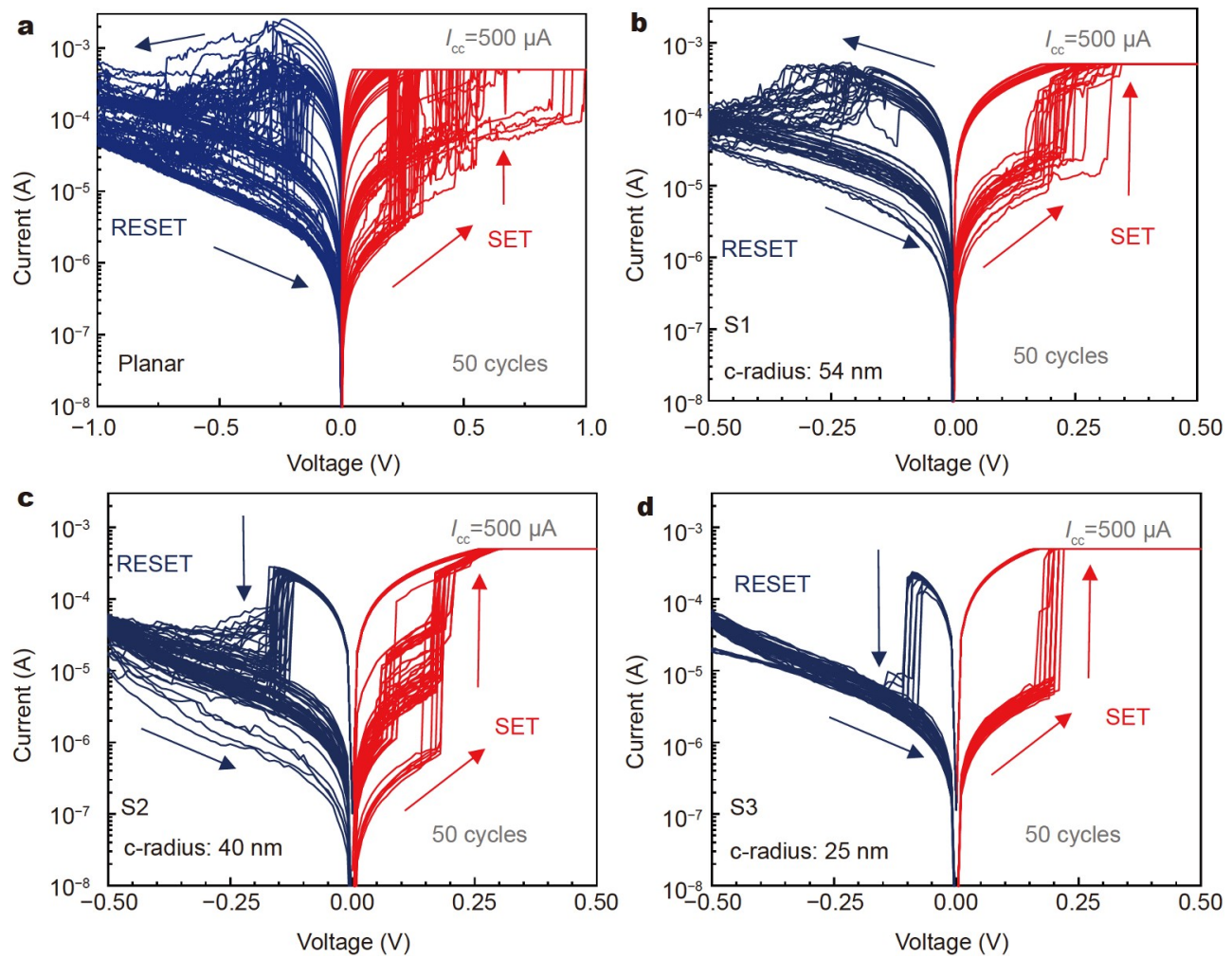

Figure 2 Typical cycle-to-cycle $I-V$ curves of the (a) planar, (b) S1, (c) S2, and (d) S3 RRAM devices. Fifty consecutive switching cycles from the same devices are shown for all the subulate and planar devices. The subulate RRAM devices exhibit superior uniformity. 
the subulate structure especially with small c-radius brings obvious improvement in $I-V$ performance of the RRAM device and dominates the evolution of RESET behavior by the feasible way of concentrating the local electric field in the TR. In addition, the S3 also exhibits highly uniform volatile-switching behavior with SET compliance current of $100 \mu \mathrm{A}$ (Fig. S3).

To provide an intuitive comparison of the switching parameters of the subulate (S1, S2, S3) and planar RRAM devices, the distributions of $V_{\mathrm{SET}}, V_{\mathrm{RESET}}$, HRS, and LRS values extracted from the abovementioned switching cycles were analyzed (Fig. 3). LRS and HRS were read at $0.1 \mathrm{~V}$ after SET and RESET operation. Compared with the planar device, the subulate devices possess tighter dispersions and lower values for both $V_{\text {SET }}$ and $V_{\text {RESET }}$ as shown in the cumulative probability in Fig. 3a-d. More details can be seen in the box plots in Fig. S4, where the $V_{\mathrm{SET}}$ values of $\mathrm{S} 1, \mathrm{~S} 2$, and $\mathrm{S} 3$ are confined to the ranges of $0.15-0.30,0.15-0.21$, and $0.17-0.20 \mathrm{~V}$, while the $V_{\text {RESET }}$ values of these devices are confined to $-0.38--0.28,-0.13--0.09$, and $-0.13-0.04 \mathrm{~V}$, respectively. Notably, low operating voltages can reduce the degradation probability and facilitate the integration with a peripheral circuit [39]. The distribution of LRS, rather than HRS, follows the same trend in that the subulate device with a smaller c-radius exhibits a tighter and smaller LRS. HRS of the subulate devices is relatively larger than the planer ones and increases with the shrinkage of the c-radius. The increased HRS should be derived from the suppression of the subulate structure upon the overinjection of cations into the RS layer [26,27]. Therefore, besides the improvement of the cycleto-cycle switching uniformity, the switching window (HRS/LRS) can be effectively enlarged with the decrement of the c-radius.

The device-to-device uniformity has also been investigated to verify the effectiveness of the wafer-scale SSA strategy. The $V_{\mathrm{SET}}$, $V_{\text {RESET }}$, HRS, and LRS distributions from 250 cycles of 10 stochastically selected devices for each configuration (S1, S2, S3) were measured and extracted. As shown in Fig. 4, the S3 device with the smallest c-radius shows the most excellent uniformity and has the largest switching window among all the subulate devices. To evaluate the impact of the SSA on the RS reliability,
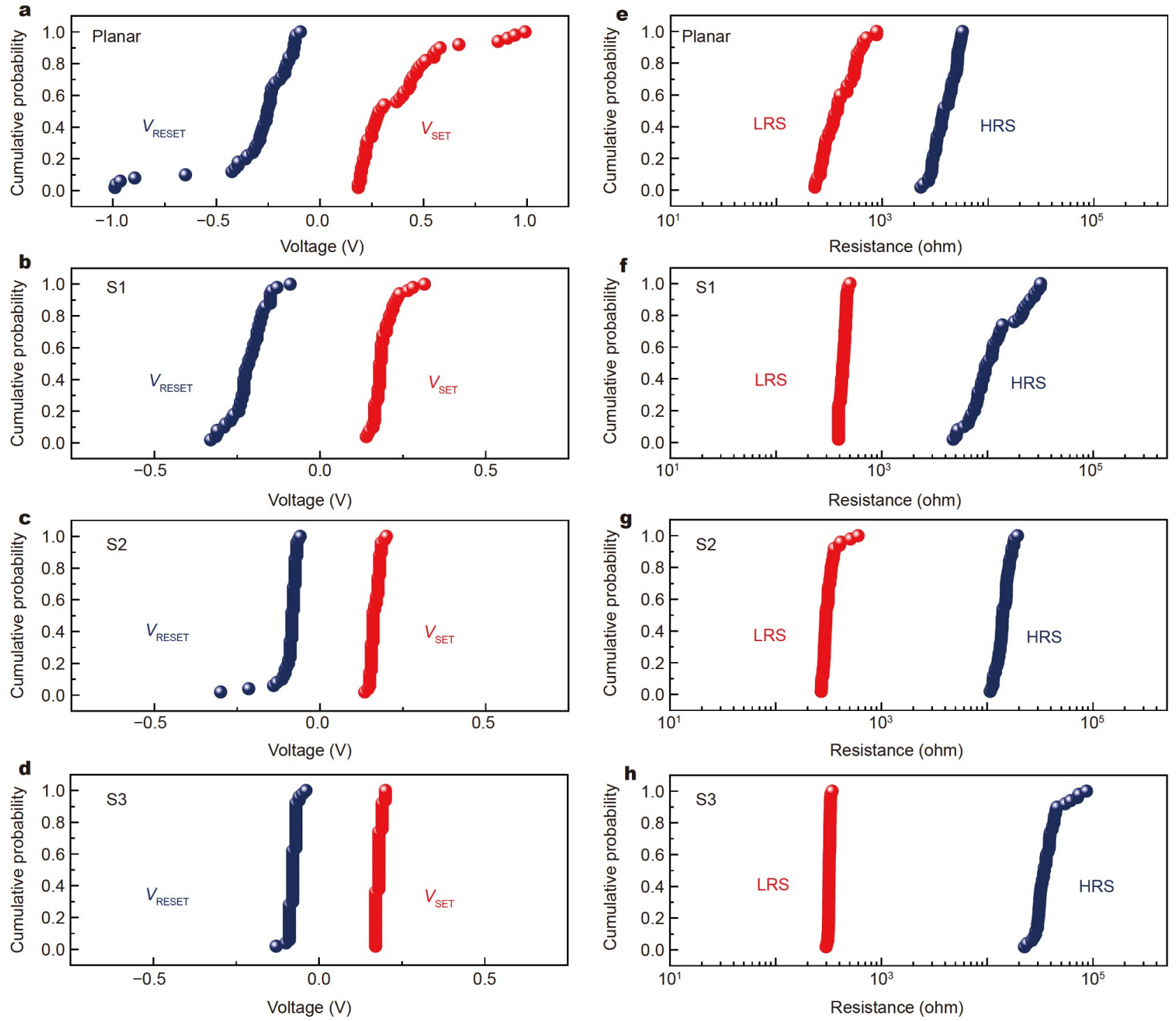

Figure 3 Distributions of cycle-to-cycle switching parameters. Cumulative probabilities of switching voltages ( $V_{\mathrm{SET}}$ and $V_{\mathrm{RESET}}$ ) and resistances (LRS and HRS) of (a, e) planar, (b, f) S1, (c, g) S2, and (d, h) S3 devices. 

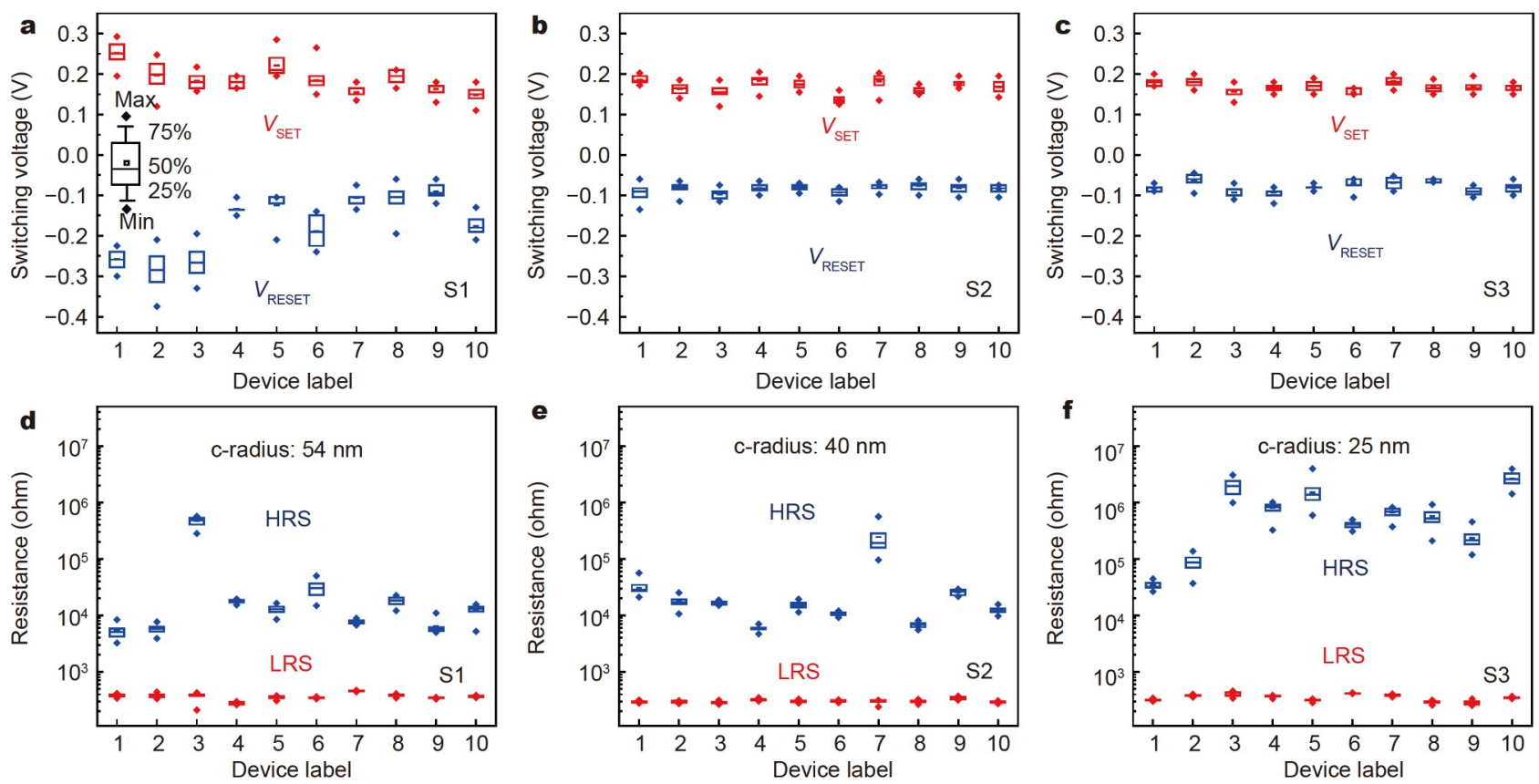

Figure 4 Device-to-device variation. Distributions of (a-c) $V_{\mathrm{SET}} \& V_{\mathrm{RESET}}$, and (d-f) HRS \& LRS from 250 cycles of 10 stochastically selected devices of S1, S2, and S3.

the retention, endurance, and switching speed of the subulate $\mathrm{Pt} /$ $\mathrm{ZrO}_{2} / \mathrm{Ag}$ devices were also measured and discussed. From the retention measurement shown in Fig. S5, the HRS, LRS, and HRS/LRS ratio of S3 are well maintained for $10^{4} \mathrm{~s}$ without obvious degradation, while S2 breaks down at nearly $10^{4} \mathrm{~s}$ and S1 breaks down at approximately $10^{3} \mathrm{~s}$. In addition, the S3 device maintains an HRS/LRS ratio above $10^{2}$ during 1000 consecutive switching cycles, as shown in Fig. S6a, indicating the considerable endurance of the S3 device with the smallest c-radius. The switching time of the device was evaluated based on voltage-time $(V-t)$ and current-time $(I-t)$ synchronous curves measured under the pulse mode, as shown in Fig. S6b, where both the LRS and HRS switch to their opposite state under a 1-ms negative RESET or positive SET pulse. As marked by the black dashed circles, the OFF and ON times are determined to be within $1 \mu$ s. Even the Ag-based RRAM is not competitive in retention, switching speed, and endurance under the pulse mode, the effectiveness of SSA strategy in improving uniformity demonstrates its expansibility towards other RRAM paradigms.

To determine the effects of the SSA strategy on CF formation, the $\mathrm{Pt} / \mathrm{ZrO}_{2} / \mathrm{Ag}$ subulate device after the SET process was investigated by TEM and EDS (detailed in the EXPERIMENT SECTION), which have been widely used to visually characterize CFs $[8,9,40-43]$. Fig. 5a shows a cross-sectional HRTEM image of the TR in the subulate RRAM device, where two potential cone-like CFs are observed in the TR to connect the Ag and $\mathrm{Pt}$ electrodes, as indicated by the red arrows. The chemical components of the CFs were studied by EDS in scanning TEM (STEM) mode. As shown in the Ag elemental mapping result in Fig. 5b, the intensity of the Ag signal in the RS layer is highly concentrated in the TR, implying that the chemical compositions of the CFs in the LRS device are mainly dominated by metallic Ag element. No trace of CFs was found in the slope region beyond the TR of the subulate RRAM device, which has been confirmed by the HRTEM image and EDS mapping along all the slope regions. The representative HRTEM and EDS results from a typical slope region are shown in Fig. $5 c$, d. The corresponding EDS element line scan profiles of positions 1-3 are shown in Fig. 5e-g, where Ag element of lines 1 and 2 rather than line 3 present obvious permeation into the $\mathrm{ZrO}_{2}$ layer near the TR. These results confirm that the wafer-scale SSA strategy facilitates the formation of a few CFs in the TR of subulate RRAM devices.

To explore the underlying relationship between the RRAM performance and the device structure morphology, the electric field distribution was simulated using the SILVACO ${ }^{\mathrm{TM}}$ tool. In this simulation, we modeled a planar RRAM device and three subulate RRAM devices with different $c$-radii in the TR (Fig. 5h, i and Fig. S7). The isosceles triangle shape of the TR is an approximate treatment for simulation convenience. In the RS layer of the planar device (Fig. 5h), the electric field exhibits uniform characteristics, while in the subulate RRAM devices, the TR shows an obviously enhanced electric field intensity relative to the other regions. Consequently, the active switching region for $\mathrm{CF}$ formation is the TR of the subulate structure, as illustrated in Fig. 5i. Fig. 5j depicts the maximum electric field $\left(E_{\mathrm{MAX}}\right)$ as a function of the apex angle, where $E_{\mathrm{MAX}}$ decreases as the degree increases (the detailed simulation parameters are displayed in Table S1). As a result, a small c-radius promotes the electric field enhancement effect most among all subulate devices, consistent with previous reports on point discharge theory [44-48]. The electric field enhancement effect suppresses the CF randomness and accounts for the superior uniformity of the S3 devices with the smallest c-radius.

\section{CONCLUSIONS}

In conclusion, we proposed a low-cost, CMOS-compatible, nanoscale-controllable, and wafer-scale SSA strategy to enhance RRAM uniformity for large-scale integration. The c-radius of $\mathrm{Si}$ subulate can be well modulated by controlling the etching time. 

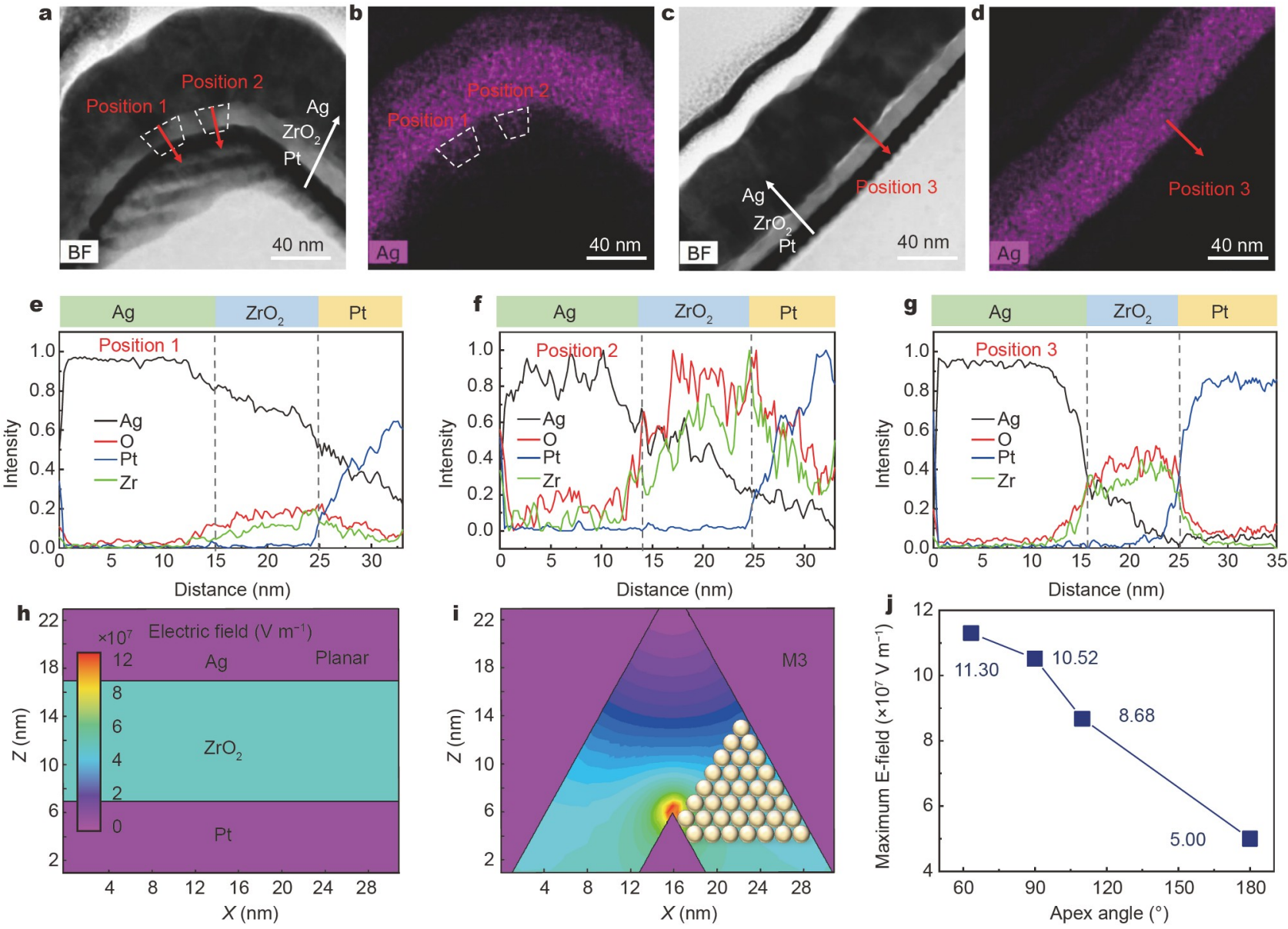

Figure 5 Formation of CF at the TR. (a) Cross-sectional HRTEM image of the TR of the subulate $\mathrm{Ag} / \mathrm{ZrO} / 2 / \mathrm{Pt}$ RRAM device at its LRS. Two CFs (positions 1 and 2) have been observed in the TR of the subulate device. (b) EDS mapping of the Ag element in the TR. (c) Cross-sectional HRTEM image of the slope beyond the TR of the subulate RRAM device and (d) the corresponding Ag element EDS mapping. (e-g) EDS spectra of the three positions as indicated in (a) and (c) by the red arrows. Position 3 is a typical region of the slope plane and no CFs are formed beyond the TR. (h, i) Simulation of the electric field distributions for the planar and proposed subulate RRAM devices with $63.3^{\circ}$ apex angle by the SILVACO ${ }^{\mathrm{TM}}$ simulation tool. CFs tend to form at the position with a localized electric field enhancement. (j) Maximum electric field as a function of the apex angle during the simulation.

Even though limited by the lithography technology, the smallest c-radius of $25 \mathrm{~nm}$ has been realized. Owing to the electric field enhancement effect, the SSA structure induces a quasi-single CF or a few CFs formed in the TR of each device, as has been verified by HRTEM and EDS characterization. The decrease in the randomness of the CF formation and rupture in the TR during SET/RESET processes promotes the RRAM device performance. Compared with the conventional planar RRAM device, the subulate devices exhibit admirable cycle-to-cycle and device-to-device uniformity and excellent stability. Additionally, with the decrement of the $\mathrm{Si}$ subulate c-radius, the device exhibits minified switching voltage and enlarged switching window. This CMOS-compatible method of engineering waferscale Si-based subulate devices, which greatly reduces the dispersion of RS parameters, provides an effective way to obtain reliable and uniform devices for the commercialization of RRAM.

Received 24 November 2021; accepted 31 December 2021; published online 14 February 2022

1 Meijer GI. Who wins the nonvolatile memory race? Science, 2008, 319: $1625-1626$
2 Li Y, Long S, Liu Q, et al. Resistive switching performance improvement via modulating nanoscale conductive filament, involving the application of two-dimensional layered materials. Small, 2017, 13: 1604306

3 Hui F, Grustan-Gutierrez E, Long S, et al. Graphene and related materials for resistive random access memories. Adv Electron Mater, 2017, 3: 1600195

4 Waser R, Aono M. Nanoionics-based resistive switching memories. Nat Mater, 2007, 6: 833-840

5 Kim KH, Gaba S, Wheeler D, et al. A functional hybrid memristor crossbar-array/CMOS system for data storage and neuromorphic applications. Nano Lett, 2012, 12: 389-395

6 Nagashima K, Yanagida T, Oka K, et al. Intrinsic mechanisms of memristive switching. Nano Lett, 2011, 11: 2114-2118

7 Waser R, Dittmann R, Staikov G, et al. Redox-based resistive switching memories-nanoionic mechanisms, prospects, and challenges. Adv Mater, 2009, 21: 2632-2663

8 Kwon DH, Kim KM, Jang JH, et al. Atomic structure of conducting nanofilaments in $\mathrm{TiO}_{2}$ resistive switching memory. Nat Nanotech, 2010, 5: 148-153

9 Yang JJ, Pickett MD, Li X, et al. Memristive switching mechanism for metal/oxide/metal nanodevices. Nat Nanotech, 2008, 3: 429-433

10 Janousch M, Meijer G, Staub U, et al. Role of oxygen vacancies in $\mathrm{Cr}$ doped $\mathrm{SrTiO}_{3}$ for resistance-change memory. Adv Mater, 2007, 19: 2232-2235 
11 Guan X, Yu S, Wong HSP. On the switching parameter variation of metal-oxide RRAM-Part I: Physical modeling and simulation methodology. IEEE Trans Electron Devices, 2012, 59: 1172-1182

12 Luo WC, Liu JC, Lin YC, et al. Statistical model and rapid prediction of RRAM SET speed-disturb dilemma. IEEE Trans Electron Devices, 2013, 60: $3760-3766$

13 Wang G, Long S, Yu Z, et al. Improving resistance uniformity and endurance of resistive switching memory by accurately controlling the stress time of pulse program operation. Appl Phys Lett, 2015, 106: 092103

14 Long S, Perniola L, Cagli C, et al. Voltage and power-controlled regimes in the progressive unipolar reset transition of $\mathrm{HfO}_{2}$-based RRAM. Sci Rep, 2013, 3: 2929

15 Long S, Lian X, Ye T, et al. Cycle-to-cycle intrinsic reset statistics in $\mathrm{HfO}_{2}$-based unipolar RRAM devices. IEEE Electron Device Lett, 2013, 34: 623-625

16 Wong HSP, Lee HY, Yu S, et al. Metal-oxide RRAM. Proc IEEE, 2012 100: $1951-1970$

17 Yang JJ, Strukov DB, Stewart DR. Memristive devices for computing. Nat Nanotech, 2013, 8: 13-24

18 Yoon JH, Han JH, Jung JS, et al. Highly improved uniformity in the resistive switching parameters of $\mathrm{TiO}_{2}$ thin films by inserting $\mathrm{Ru}$ nanodots. Adv Mater, 2013, 25: 1987-1992

19 Chen L, Gou HY, Sun QQ, et al. Enhancement of resistive switching characteristics in $\mathrm{Al}_{2} \mathrm{O}_{3}$-based $\mathrm{RRAM}$ with embedded ruthenium nanocrystals. IEEE Electron Device Lett, 2011, 32: 794-796

20 Guan W, Long S, Jia R, et al. Nonvolatile resistive switching memory utilizing gold nanocrystals embedded in zirconium oxide. Appl Phys Lett, 2007, 91: 062111

21 Ryu SW, Ahn YB, Kim HJ, et al. Ti-electrode effects of NiO based resistive switching memory with $\mathrm{Ni}$ insertion layer. Appl Phys Lett, 2012, 100: 133502

22 Lin $\mathrm{CY}$, Lin $\mathrm{MH}$, Wu MC, et al. Improvement of resistive switching characteristics in $\mathrm{SrZrO}_{3}$ thin films with embedded $\mathrm{Cr}$ layer. IEEE Electron Device Lett, 2008, 29: 1108-1111

23 Lv H, Wan H, Tang T. Improvement of resistive switching uniformity by introducing a thin GST interface layer. IEEE Electron Device Lett, 2010, 31: 978-980

24 Zhang $\mathrm{H}$, Liu L, Gao B, et al. Gd-doping effect on performance of $\mathrm{HfO}_{2}$ based resistive switching memory devices using implantation approach. Appl Phys Lett, 2011, 98: 042105

25 Yoon J, Choi $\mathrm{H}$, Lee D, et al. Excellent switching uniformity of $\mathrm{Cu}$ doped $\mathrm{MoO}_{x} / \mathrm{GdO}_{x}$ bilayer for nonvolatile memory applications. IEEE Electron Device Lett, 2009, 30: 457-459

26 Zhao X, Ma J, Xiao X, et al. Breaking the current-retention dilemma in cation-based resistive switching devices utilizing graphene with controlled defects. Adv Mater, 2018, 30: 1705193

27 Zhao X, Liu S, Niu J, et al. Confining cation injection to enhance CBRAM performance by nanopore graphene layer. Small, 2017, 13: 1603948

28 Shin KY, Kim Y, Antolinez FV, et al. Controllable formation of nanofilaments in resistive memories via tip-enhanced electric fields. Adv Electron Mater, 2016, 2: 1600233

29 Kim Y, Choi H, Park HS, et al. Reliable multistate data storage with low power consumption by selective oxidation of pyramid-structured resistive memory. ACS Appl Mater Interfaces, 2017, 9: 38643-38650

30 Kim S, Jung S, Kim MH, et al. Nano-cone resistive memory for ultralow power operation. Nanotechnology, 2017, 28: 125207

31 Coluccio ML, Francardi M, Gentile F, et al. Plasmonic 3D-structures based on silver decorated nanotips for biological sensing. Opt Laser Eng, 2016, 76: 45-51

32 Li X, Zhang B, Wang B, et al. Low power and high uniformity of $\mathrm{HfO}_{x}$ based RRAM via tip-enhanced electric fields. Sci China Inf Sci, 2019, 62: 202401

33 Li W, Song X, Zhao X, et al. Design of wafer-scale uniform Au nanotip array by ion irradiation for enhanced single conductive filament resistive switching. Nano Energy, 2020, 67: 104213

34 Kim HJ, Yoon KJ, Park TH, et al. Filament shape dependent reset behavior governed by the interplay between the electric field and thermal effects in the $\mathrm{Pt} / \mathrm{TiO}_{2} / \mathrm{Cu}$ electrochemical metallization device. Adv Electron Mater, 2017, 3: 1600404

35 Celano U, Goux L, Belmonte A, et al. Understanding the dual nature of the filament dissolution in conductive bridging devices. J Phys Chem Lett, 2015, 6: 1919-1924

36 Celano U, Giammaria G, Goux L, et al. Nanoscopic structural rearrangements of the $\mathrm{Cu}$-filament in conductive-bridge memories. $\mathrm{Na}$ noscale, 2016, 8: 13915-13923

37 Berco D, Tseng TY. A numerical study of multi filament formation in metal-ion based CBRAM. AIP Adv, 2016, 6: 025212

38 Villena MA, Roldán JB, Jimenez-Molinos F, et al. A comprehensive analysis on progressive reset transitions in RRAMs. J Phys D-Appl Phys, 2014, 47: 205102

39 Jo $\mathrm{SH}$, Lu W. CMOS compatible nanoscale nonvolatile resistance switching memory. Nano Lett, 2008, 8: 392-397

40 Chen JY, Huang CW, Chiu CH, et al. Switching kinetic of VCM-based memristor: Evolution and positioning of nanofilament. Adv Mater, 2015, 27: 5028-5033

41 Yang Y, Gao P, Li L, et al. Electrochemical dynamics of nanoscale metallic inclusions in dielectrics. Nat Commun, 2014, 5: 4232

42 Liu Q, Sun J, Lv H, et al. Real-time observation on dynamic growth/ dissolution of conductive filaments in oxide-electrolyte-based ReRAM. Adv Mater, 2012, 24: 1844-1849

43 Choi SJ, Park GS, Kim KH, et al. In situ observation of voltage-induced multilevel resistive switching in solid electrolyte memory. Adv Mater, 2011, 23: 3272-3277

44 Zhang Z, Zhao Y, Zhu M. NiO films consisting of vertically aligned cone-shaped NiO rods. Appl Phys Lett, 2006, 88: 033101

45 Chang WY, Lin CA, He-Hau J, et al. Resistive switching behaviors of ZnO nanorod layers. Appl Phys Lett, 2010, 96: 242109

46 Zhu YW, Zhang HZ, Sun XC, et al. Efficient field emission from ZnO nanoneedle arrays. Appl Phys Lett, 2003, 83: 144-146

47 Spindt CA, Brodie I, Humphrey L, et al. Physical properties of thin-film field emission cathodes with molybdenum cones. J Appl Phys, 1976, 47: 5248-5263

48 Huang YC, Tsai WL, Chou CH, et al. High-performance programmable metallization cell memory with the pyramid-structured electrode. IEEE Electron Device Lett, 2013, 34: 1244-1246

Acknowledgements This work was supported by the National Natural Science Foundation of China (61925110, 61821091, 51961145110, 62004184, and 62004186), the Ministry of Science and Technology of China (2016YFA0201803, 2016YFA0203800, and 2017YFB0405603), the Key Research Program of Frontier Sciences of Chinese Academy of Sciences (QYZDY-SSW-JSC001 and QYZDB-SSW-JSC048), the Fundamental Research Funds for the Central Universities (WK2100000014 and WK2100000010), China Postdoctoral Science Foundation (2020M671895 and BX20200320), and the Opening Project of Key Laboratory of Microelectronic Devices \& Integration Technology, Institute of Microelectronics, Chinese Academy of Sciences. This work was partially carried out at the Center for Micro and Nanoscale Research and Fabrication, University of Science and Technology of China.

Author contributions Zhao $\mathrm{X}$ and Long $\mathrm{S}$ designed the project and guided the research. Zhang $\mathrm{Y}$, Zhang $\mathrm{M}$, and Liu $\mathrm{Y}$ fabricated the samples and performed the electrical switching experiments. Ma X and Zhou X simulated the electrical field distribution. Xu G assisted in the data analysis. Zhang Y and Zhao X wrote and revised the paper. All authors contributed to extensive discussions of the results.

Conflict of interest The authors declare that they have no conflict of interest.

Supplementary information Supporting data are available in the online version of the paper. 


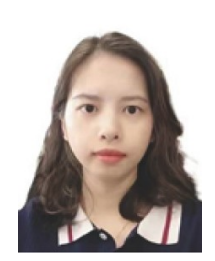

Ying Zhang is currently a doctoral student at the Institute of Microelectronics, Chinese Academy of Sciences. She received her bachelor's degree from the University of Science and Technology Beijing in 2018. Her current research focuses on the resistive random access memory.

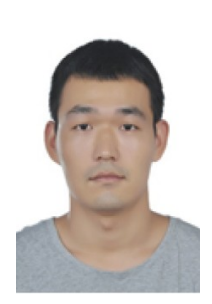

Xiaolong Zhao is currently a postdoctor at the University of Science and Technology of China, Hefei. He received his $\mathrm{PhD}$ degree from Wuhan University in 2019. His current research interest mainly focuses on photodetectors based on wide-bandgap materials, novel in-sensor computing devices, and resistive random access memory.

\section{CMOS兼容的晶圆级硅雉阵列局域化细丝形成以实 现极佳阻变存储器均一性}

张颖 ${ }^{1,2,3}$, 赵晓龙 ${ }^{2}$, 马晓兰 ${ }^{2}$, 刘宇 ${ }^{1}$, 周选择 ${ }^{2}$, 张美芸 ${ }^{1}$, 徐光伟 ${ }^{2}$, 龙世兵 ${ }^{2}$

摘要 具有高密度三维集成特点的阻变存储器(RRAM) 是下一代非易 失性存储技术的有利竞争者之一. 然而, 导电细丝形成和断裂的随机性 导致了RRAM均一性差的问题, 这严重阻碍了RRAM芯片的大规模商 用. 目前, 已有部分研究通过引入锥形结构, 减少RRAM中导电细丝形 成和断裂的随机性. 但是, 这些方法往往步骤繁琐、成本较高或分辨率 有限, 限制了这些技术的大规模推广. 本研究提出了一种CMOS兼容 的、可在纳米尺度调控的晶圆级硅雉阵列(SSA)制备方法. 该方法可制 备不同曲率半径的SSA，用于调控RRAM中的导电细丝. 高分辨率透射 电子显微镜和能量色散谱表征结果表明, SSA结构诱导器件在尖端区域 形成准单根或少量的导电细丝, 显著改善了器件转变参数的均一性. 此 外, 减小尖端区域的曲率半径可显著提升器件转变电压和高/低阻态的 分布均一性及器件阻态的保持特性等. 器件转变参数均一性的改善归 因于尖端区域内的局域电场增强效应. 本研究所提出的SSA方法具备 低成本、CMOS兼容且纳米尺度可控的特点, 为高均一性RRAM器件 的大规模集成提供了一种参考策略. 